\title{
XXXIII. \\ Ueber die Einwirkung der Centralorgane auf die Erregbarkeit der motorischen Nerven.
}

\author{
Von
}

\author{
Dr. Th. Rumpf, \\ Assistenzarzt an der elektro-therapeutischen Station in Heidelberg.
}

\begin{abstract}
Der Einführung der elektrischen Untersuchungsmethoden in die Neuropathologie haben wir im Laufe der letzten Jahre eine Reihe der interessantesten Ergebnisse zu danken gehabt. Besonders sind die Störungen in den Nerven und Muskeln bei Affection der Nerven in ihrem Verlauf oder der Ganglienzellen in den granen Vordersäulen des Rückenmarks durch die Ergebnisse der Prüfung der faradischen und speciell der galvanischen Erregbarkeit ein dankbares Feld der Untersuchung gewesen.
\end{abstract}

Diese Untersuchungen haben uns vor Allem mit Bestimmtheit zu dem Schluss geführt, dass von den Centralorganen aus eine ständige Einwirkung auf Nerven und Muskeln Statt haben muss, deren Aufgabe eine Regelung der Ernährung dieser ist, eine Einwirkung, die wir nach allen Thatsachen trophischen Centren in der grauen Substanz des Rückenmarks zuschreiben müssen.

Selbstverständlich muss diese trophischc Einwirkung auch die übrigen Gewebe treffen, wie dieses die Wachsthumshemmung an den Knochen, die Veränderung an Gelenken, Gefässen und Haut bei der spinalen Kinderlähmung zur Genüge beweisen. Aber die Deutung dieser Veränderungen ist gegen die der Lähmung und Atrophie der Muskeln leider weit zurück geblieben, was wir wohl einerseits der Schwierigkeit der Untersuchnng, andererseits der Unzugänglichkeit für das Experiment zuschreiben müssen. 
Die elektrische Untersuchung der Nerven und Mnskeln in Verbindung mit dem übrigen Befund haben dann noch zu einer Reibe weiterer Ergebnisse geführt.

So schliesst Er $b^{*}$ ) aus dem Vorkommen von centralen Lähmungen mit und ohne Atrophie und ferner von Atrophie und Entartung der Muskeln bei centralen Affectionen ohne Lähmung, bei erhaltener Erregbarkeit der zu diesen führenden Nerven, dass die motorischen Bahnen im Centralorgan bis zu einem gewissen Grade getrennt von den trophischen Apparaten verlaufen, und aus der Thatsache, dass die Muskeln allein degeneriren können, ohne dass die Nerven an dieser Degeneration Theil nehmen, dass auch für die Nerven und Muskeln getrennte trophische Centralapparate existiren.

$O b$ von diesen trophischen Centren aus besondere, von den motorischen Bahnen getrennte und für sich bestehende trophische Nerven zu diesen und den Muskeln verlaufen, lässt Erb bei dieser Frage unberührt. Ist dieses aber nicht der Fall, und die Existenz besonderer trophischer Nerven ist jedenfalls nicht erwiesen, so müssen schon vorhandenen centrifugalen Nerven die trophischen Einwirkungen zngeschrieben werdeu. Bleiben wir bei den Nerven und Muskeln, so müssen also vom Centralorgan aus ständige trophische Einwirkungen durch die motorischen Nerven diesen und den Muskeln übermittelt werden und so die Ernährung beider regeln.

Für den Nerven ist dieses allerdings nichts Besonderes. Dass ein sorgfältig von der Umgebung gelöster Nerv lange Zeit seine willkürliche und elektrische Erregbarkeit behält, sofern er nur gegen das Vertrocknen geschützt ist, lässt doch mit Sicherheit den Schluss zu, dass die Ernährung der Nerven nur von dem Centralorgan oder von der Peripherie aus, also von den Nervenendplatten in den Muskeln geschehen kann.

Das Centralorgan ist für die Eruährung der motorischen Nerven schon lange in Anspruch genommen worden. Dass ein Theil der Ernährung jedoch den Nervenendplatten in den Muskeln zufällt, hat $\mathrm{Kühn} \mathrm{e}^{* * *}$ ) durch seine Versuche mit Curare and durch Unterbindung der Muskelarterien bewiesen.

Auch die Pathologie lässt eine Ernährung von Seiten der Peripherie aus jenen Fällen schliessen, bei denen die motorische Leitung im Nerven unterbrochen ist, während der Nerv nicht degenerirt, sondern elektrisch

*) y. Ziemssen, Handbuch d. spec. Pathol. XI, Krankheiten des Rückenmarks, 2. Seite 310 und dies. Archiv Band VIII., 1. S. 216.

$* *$ ) Lehrbuch der physiol. Chemie. S. 352. 
erregbar bleibt, wenn man nicht gerade annehmen will, dass die Läsionsstelle zwar der motorischen Leitung ein Hinderniss sei, aber ständige trophische Strömungen ohne Unterbrechung passiren lasse, wogegen der eine Cmstand vielleicht zur Genüge spricht, dass trotz dieses Erhaltenbleibens der Nerven Atrophie und Entartung der Muskeln eintreten kann.

Aber auch für die Muskeln hat die Anschauung einer durch die motorischen Nerven übermittelten ständigen trophischen Einwirkung nichts ganz Unwahrscheinliches.

Denn die Veränderungen der elektrischen Erregbarkeit der motorischen Nerven bei Zuständen, bei denen die Nerven nicht gelähmt und degenerirt, dagegen die Muskeln in der einen oder andern Weise trophisch gestört sind, und das Parallelgehen dieser Veränderungen mit den Störungen in den Muskeln geben wohl der Ansicht Raum, dass ausser der motorischen Leitung noch andere Einflüsse durch die motorischen Nerven übermittelt werden, deren Veränderung sich möglicher Weise durch Modification der elektrischen Erregbarkeit ausdrückt.

Allerdings sind diese Modificationen der elektrischen Erregbarkeit noch lange nicht genügend untersucht, so dass von Seiten der Pathologie eine Erklärung dieser wechselnden Zustände bis jetzt nicht erfolgen kounte.

Von Seiten der experimentellen Physiologie sind die Schwierigkeiten ziemlich bedeutend. Die trophischen Störungen in den Nerven and Maskeln nach Trennung der Nerven in ihrem Verlanf wurden anch durch Experimente am Thiere vollständig bestätigt. Ob aber diese trophischen Störungen mit dem Wegfall der willkürlichen Bewegung oder mit dem einer spinalen Einwirkung zusammenhängen, war experimentell nicht zu entscheiden. Dass mit dem Wegfall der willkürlichen Bewegung noch lange keine Entartung der Muskeln auftritt, ist ja klinisch bekannt. Aber eine ständige Einwirkung des Rückenmarks auf die willkürlichen Muskeln, nnd etwas anderes heisst doch der vielfach discutirte Muskeltonus nicht, war wenigstens bis jetzt insofern nicht zu erweisen, als es keinem der verschiedensten Forscher gelungen ist, eine ständige, von der willkürlichen unabhängige, Contraction der Muskeln zu erweisen. Und in dieser hatten doch ziemlich sämmtliche Forscher den Ausdruck des Muskeltonus erblicken zu müssen geglaubt. Anders allerdings Cyon*), der den-

*) Berichte über d. Verhandlung. d. Kgl. Sächs. Gesellschaft d. Wissenschaft. Math.-physic. Klasse XVII., 1865. 
selben in einer Erhöhung der Erregbarkeit der motorischen Bahnen suchte. Indem er von seiner, jedoch nichts weniger als erwiesenen Hypothese ausging, dass die Coordination der Bewegungen durch einen Reflexact zu Stande komme, wodurch die Ataxie bei der Tabes in einem Wegfallen der sensibeln Erregungeu ihre Erklärung fände, prüfte er die Erregbarkeit der motorischen Wurzeln vor und nach der Durchschneidung der sensibeln und glaubte ein Sinken der Erregbarkeit nach der Durchschneidung dieser gefunden zu haben. Seine Resultate wurden zwar von Guttmann ${ }^{*}$ bestätigt, jedoch von v. Bezold und Uspensky**) auf Versnchsfehler zurückgeführt und auch Steinmann's***) Resultate, der unter Cyon's Leitung im physiologischen Institut zu St. Petersburg mit exacteren Messungsmethoden arbeitete und für die Cyon'sche Ansicht von der Beeinflussung der Erregbarkeit der vordern Wurzeln durch die hintern eintrat, konnte Heidenhainf) in keiner Weise bestätigen.

Im Uebrigen ist der mit dem Centralorgan in Verbindung stehende Nerv wohl selten Gegenstand der elektrischen Lntersuchung gewesen. Jedenfalls ist aber für ihn kein abweichendes Zuckungsgesetz in Anspruch genommen worden und auch von Fleisehl, $\dagger \dagger$ ) der in letzter Zeit allerdings bis jetzt nur mit secundären Strömen das Verhalten des mit dem Centralorgan in Verbindung stehenden Nerven untersucht hat, findet $z$ wischen diesem und dem getrennten nur die eine Differenz, dass der von ihm sogenannte Aequator des Nerven, an dem aufsteigende und absteigende Ströme von gleicher Stärke gleiche Zuckungen auslösen, nach der Durchschneidung nach abwärts wandert. Dieser sogenannte Aequator unterscheidet sich von dem obern und untern Abschnitt des Nerven dadurch, dass im obern absteigende und im untern aufsteigende Ströme beträchtlich früher Zuckungen auslösen als die jeweilig entgegensetzten.

Zur Eliminirung einer Fehlerquelle, die die Untersuchungen an dem mit dem Centralorgan verbundenen Nerven und Muskel allerdings stets bedeutend gehindert hat, nämlich die durch Reizung der sensibeln Nerven etwa entstehenden Reflexzuckungen auzuschalten, be-

*) Med. Centralblatt 1867. No. 44. Heft III.

**) Gescheidlen, Studien des physiol. Laboratoriums zu Würzburg.

***) Bulletin de l'Acadèmie de St. Petersbourg: Ueber den Tonas der willkürlichen Muskeln.

†) Pflüger's Archiv. IV. Jahrgang. S. 435.

††) Sitzungsberichte d. K. Akademie d. Wissensch. Jahrgang 1876. 
diente sich von Fleischl hauptsächlich des Chlorals, das nach seiner Angabe die reflectorischen Zuckungen mit derselben Exactheit ausschliesst, wie das jedenfalls sicherste, wenn auch sonst nicht unbedenkliche Mittel, nämlich die Durchschneidung der sensibeln Wurzeln.

Meine eigenen Versuche, die ich durch die Güte des Herrn Geheimrath Kühne im physiologischen Institut zu Heidelberg anstellen konnte, wurden Anfangs mehr in Rücksicht auf das Zuckungsgesetz am Menschen angestellt, ein Unstand, der wohl für die anfänglich vorhandenen und später eliminirten Fehlerquellen als Entschuldigung dienen wird. Bis jetzt habe ich mich nur des aufsteigenden Stromes bedient. Es liegt das daran, dass gerade bei seiner Benutzung an dem mit dem Centralorgan verbundenen Nerven, sich wesentliche Differenzen zeigten.

Und da die Resultate dieser physiologischen Untersuchungen zu einer Reihe weiterer Untersuchungen in der Pathologie Veranlassung wurden, und auch hier vollständige Bestätigung zu finden scheinen, so zögere ich nicht, dieselben zu veröffentlichen.

Ich bediente mich, wie schon bemerkt, zu meinen Untersuchungen des constanten Stromes und zwar wurde für gewöhnlich nur ein Daniel'sches Element als Stromquelle benutzt. Die Nebenschliessung bestand in einem du Bois schen Rheochord. Von den Klemmen des Rheochords liefen die Drähte für den Nerven durch einen Stromwender und von da der eine direct zu seiner Elektrode, der andere zu einem Schlüssel, von dem aus die Leitung dann zur zweiten Elektrode ging.

Als Elelitroden benutzte ich den ersten Theil meiner Untersuchungen gut polirte Zinkdrähte in Hülsen von Guttapercha, die nur eine breite Oeffnung zur Berührung der Drähte mit dem Nerv hatten. Anfangs wurden die Elektroden unter den frei präparirten Nervus ischiadicus des Frosches geschoben, ein Verfahren, das ich zur Eliminirung von Fehlerquellen später besonders bei den Versuchen mit unpolisirbaren Elektroden dahin änderte, dass der Oberschenkel des Frosches amputirt wurde und nur der Nervus ischiadicus die Verbindung zwischen den Centralorganen und der Muskulatur des Unterschenkels herstellte.

Wollte ich nun einen Vergleich ziehen zwischen den Zuckungsminima bei Schliessung und Oeffnung des Stromes an dem mit dem Centralorgan verbundenen Nerven und den Resultaten am durchtrennten, so musste ich denselben Nerven prüfen erst in Verbindung. mit dem Centralorgan und dann nach Aufhebung der Leitung von diesem zu dem geprüften Theil des Nerven.

Hatte ich nun den Nerven sehr sorgfältig präparirt und liess einen a ufsteigenden Strom hindurchgehn, während im Rheochord alle Widerstände ausgeschaltet waren, so trat meist schon nach kurzer Verschiebung des Schlittens um 5-30 Centimeter bei Schliessung des Stromes Zuckung im Gastrocnemius ein. Oeffnete ich nun den Strom nach der jedesmaligen Schliessung sofort, so erfolgte in einer Reihe von Versuchen schon nach kurzer 
weiterer Vorschiebung des Schlittens, biswoilen auch alsbald Oeffnungszuckung.

In einer andern sehr grossen Reihe von Fällen fand das aber nicht statt. Ich schaltete zunächst durch Verschieben des Schlittens bis zum Ende des Rheochord's den Widerstand einer Einheit ein: es erfolgte keine Oeffnungszuckung. So schaltete ich nach und nach die gesammten Widerstände der Nebenschliessung ein, so dass nunmehr ziemlich die gesammte Stärke eines Elements beim Schliessen des Strom's durch den Nerven hindnrchging. In einzeln Fällen erfolgte auch jetzt noch keine 0effnungszuckung, sondern trat erst auf, nachdem der Strom einige Secunden durch den Nerv geschlossen gewesen war; in andern Fällen trat die Oeffnungszuckung: schon ein, als noch nicht die ganze Stärke eines Elementes durch den Nervhindurchging, sondern noch Widerstände im Reochord ansgeschaltet waren.

Noch Eines muss ich hier erwähnen, weil es mir gleichfalls nicht unwichtig erscheint. Bisweilen trat Oeffnungszuckung bei noch ganz schwachem Strom ein, oft bei Verschieben des Schlittens noch nicht um den Widerstand einer Einheit, sobald nur der Strom eine Zeit lang geschlossen gewesen war. Liess ich aber nunmehr dem Nerven etwas Zeit, wartete ich eine halbe bis ganze Minute bis zur abermaligen Schliessung und Oeffnung des Stromes, so trat bei vielen Untersuchungen keine Oeffnungszuckung auf und ich konnte sogar noch die gesammten Widerstände des Rheochord's einschalten: die 0 effnungszuokung fehlte, wie in den andern vorher erwähnten Fällen bei einfacher Schliessung und Oeffnung des Stroms und trat erst nach länger fliessenden Strömen auf. Dieses am Nerv-Muskelpräparat von mir nie beobachtete Verhalten glaubte ich entschieden anf eine Ein wirkung des Centralorgans zurückführen zu müssen.

Das frühzeitige Auftreten der Oeffnungszuckung in andern Fällen konnte ja leicht in einer Verletzung des Nerven bei der Operation seinen Grund haben. Hatte doch Westpha ${ }^{*}$ ) erst vor Kurzem gezeigt, dass schon durch leichtes Anziehen des Nerven vermittels eines Häkchens die Leitung unterbrochen werden kann, indem auf Beklopfen dor Sehne das von ihm sogenannte Unterschenkelphänomen, der Sehnenreflex Erb's in dem zugehörigen Muskel nicht mehr auftrat.

Indem ich nun bei meinen Versuchen die Sehne des Gastrocnemius auf ihre Reflexerregbarkeit prüfte, zeigte sich, dass in keinem Falle des Fehlens der Sehnenreflexe die von mir vorher erwähnte geringe Erregbarkeit für Oeffnungen des Stromes eintrat, und in allen Fällen, in denen die Muskeln mit einer Zusammenziehung auf die Sehnenreizung antworteten, die Deffnungszuckung erst spät auftrat. Allerdings ist damit kein Beweis geliefert, dass dieses Fehlen der Oeffnungszuckung auf eine Wirkung der Centralorgane zurückgeführt werden muss, wenn es auch an Wahrscheinlichkeit dadurch gewinnt.

Es musste sich doch bei allen diesen Versuchen zunächst fragen, wie

*) Dieses Archir, VII. S. 666 . 
der Nerv reagirt, nachdem eine Einwirkung der Centralorgane ausgeschlossen werden kann. Das einfachste Mittel war natürlich die 'Trennung des Nerven oberhalb der Elektroden.

Prïfte ich nun, nachdem ich eine halbe bis eine Minute nach der Durchschneidung hatte vorïber gehen lassen, so trat die Oeffnungszuckung entwe derfastgleichzeitig oder kurz nach der Schliessungszuckung auf. Die Schliessungszuckung wurde durch die Durehschneidang nicht modificirt.

Dasselbe Resulat erhielt ich, wenn ich statt der Durchschneidung des Nerven eine Zerstörung des Rïckenmarks vornahm. Abtragung des Kopfes allein hatte keinen Einfluss.

Aber gëgen beide Operationsverfahren liessen sich Einwände erheben. Es gelang selten den Nerven zu durchtrennen, ohne dass eine Zuckung ausgelöst wurde, und mit der Zerstörung des Rückenmarks waren immer Zuckungen verbunden. Es wäre also immerhin möglich gewesen, dass die frühzeitige Oeffnungszuckung nicht dem Ausfall einer vom Rückenmark ausgehenden Erregung ilhre Entstehung verdankte, sondern vielmehr einer durch die Durchtrennung des Nerven oder die Zerstörung des Rückenmarks gesetzten Reizung. Allerdings schien diese Ansicht keineswegs sehr wahrscheinlich. Hatten doch Pflüger und die übrigen Forscher bei Feststellung des Zuckungsgesetzes die Durchschneidung des Nerven ganz ausser Acht gelassen. Aber berücksichtigt musste dieser Einwand immerhin werden.

Herr Geheimrath Kühne rieth mir, mich zur Trennung des Nerven des Ammoniaks zu bedienen, das nach seiner Erfahrung die Trennung ohne irgend welchen Reiz bewirkte. Das war allerdings der Fall.

Liess ich einen Tropfen Ammoniak auf einen Nerven fallen, so war ohne jede Reizungserscheinung die Leitung nach 30 Sekunden aufgehoben. Bewerkstelligte ich nunmehr die Trennung mit Ammoniak möglichst weit dem Centrum zu, nachdem ich das Fehlen der 0effnungszuckung constatirt hatte, und reizte nach $30-40$ Sekunden, so trat jetzt die Oeffnungszuckung kurz nach der Schliessungszuckung auf.

Wartete ich aber etwas länger, so trat plötzlich nur Oeffungszuckung auf; die Schliessungszuckung war nur noch mit stärikern und allmählich immer stärkern Strömen zu erzielen. Es konnte dieses nur darin seinen Grund haben, dass das Ammoniak in dem Nerven weiter kriechend die Stelle erreichte, an der die eine Elektrode, die Kathode, dem Nerven anlag, und so dio Schliessungszuckung beeinflusste.

Ich war somit wieder auf die Durchschneidung des Nerven angewiesen, modificirte aber dieses Verfahren dadurch, dass ich eine zweimalige Durchsehneidung des Nerven vornahm, und nachdem ich sodann wieder je eine halbe oder ganze Minute bis zur abermaligen Reizung gewartet hatte, untersuchte, $a b$ die zweite Durchschneidung einen Einfluss auf die Oeffnungszuckung des aufsteigenden Stromes ausübte. Dieses war nicht der Fall.

Bei diesen letzten Untersuchungen hatte ich mich stets der unpolari- 
sirbaren Elektroden bedient und dabei den Frosch so präparirt, dass der Nerv die einzige Verbindung zwischen dem Frosch und dem Schenkel war.

Hier war allerdings die Prüfung, ob der Nerv unverletzt und leitungsfähig, schwieriger, da von den für gewöhnlich reflectorisch zuckenden Musheln die Adductoren des Oberschenkels zerschnitten und getrennt und die Wadenmuskeln durch Verschiebung ihrer Ansatzstellen vollständig erschlafft waren.

Aber bald zeigte sich, dass trotzdem noch von der Gastrocnemiussehne Reflexe hervorgerufen werden konnten, sobald man uur durch Hyperextension des amputirten Oberschenkelknochens im Kniegelenk die Sehne etwas spannte.

Ueber die Resnltate bei Anwendung der unpolarisirbaren Elektroden an dem wie oben beschrieben präparirten Nerven brauche ich nichts weiter hinzuzufügen. Sie waren vollständig dieselben. Um aber vor jedem Irrthum sicher zu sein, wurden die Versuche noch in der verschiedensten Weise modificirt.

Zunächst rieth mir Herr Geheimrath Kühn ne die Prüfung nach Anwendung einer Kältemischung anf die Wirbelsäule zu machen. Ich neigte in dem Glauben, dass das Rückenmark dadurch in seiner Thätigkeit beeinträchtigt werde, der Ansicht zu, dass in Folge davon die Oeffnungszuckung sehr früh eintreten werde. Aber gerade das Gegentheil war der Fall.

Haite eine Kältemischung unter der Rückenhaut des Frosches direct auf der Wirbelsäule eine viertel oder eine halbe Stunde gelegen, und präfte ich mit dem aufsteigenden Strom, so trat nicht nur die Oeffnungszuckung nicht früher, sondern entschieden noch später auf. Ich konnte in einzelnen Fällen zwei Elemente einscbalten, ich konnte den Strom einige Sekunden geschlossen lassen, die Oeffnungszuckung fehlte, während dieselbe kurz nach derDurehschneidung bei den geringsten Stromstärken vorhanden war. Die Reflexe waren hierbei deutlich vorhanden. *)

Der Nerv wurde bei diesen Versuchen von der Einwirkung der Kältemischung so weit als möglich freigehalten.

Dasselbe Resultat erhielt ich bei einer weiteren Versuchsreihe.

Es wurde hierbei der Frosch so präparirt, dass nichts mehr vorhanden war, als der Kopf, đie Wirbelsäule und die untern Extremitäten. Zunächst zeigte die Prüfung der Reflexe, dass dieselben entschieden erhöht waren.

Hatte ich nun rasch präparirt, so fehlte auch hier die Oeffaungszuckung bei verhältnissmässig hohen Stromstärken, aber sie trat auch nach etwa 20 Minuten an dem nicht durchschnittenen Nerven auf und übte dann die Durchschneidung keinen weitern Einfluss.

Es bleibt mir nun noch übrig einige bisher nicht beachtete Einwürfe zu. berücksichtigen.

*) Dass durch Einwirkung einer Kältemisehung auf das Rückenmark die Reflexe ausserordentlich verlangsamt werden und die Zuckungen e inen trägen tetanischen Charakter annehmen, hat, wie ich höre, Rosenthal auf der letzten Naturforscherversammlung schon hervorgehoben. 
Bei einer grössern Anzahl meiner Versuche hatte ich zur Eliminirung der Reflexzuckungen nichts angewendet. Es traten bei dem lebenden Frosch in dem zur Reizung benutzten Gastrocnemius Reflexzuckungen anf, aber grade am wenigsten direct bei Schliessung und Oeffnung des Stromes, und ich glaubte eine schon vielfach erwähnte Thatsache hier bestätigt za sehen, dass starke sensible Reize und besonders elektrische reflexhemmend wirken. Dann aber konnte die einzige Differenz des mit dem Centralorgan verbundenen Nerven vom getrennten, das Fehlen der Oeffnungszuck ung, nicht durch Reflexe bedingt sein.

Doch benutzte ich in einer grossen Reihe meiner letaten Untersuchungen das Chloralhydrat und fand auch bei Anwendung dieses meine früheren Resultate bestätigt.

Schon vorher batte ich mich zur Ausschaltung der Reflexe und noch aus einem weitern Grunde der Durchschnoidung der sensibeln Wurzeln bedient. Wenn ich auf diese Versuche näher eingehe, so geschieht das, damit keine Verwechselung mit den Resultaten Cyon's eintritt, als sei die von mir gefundene Differenz durch eine Wirliung der sensibeln Wurzeln bedingt. Einem Frosch wurden die sensibeln Wurzeln der einen Seite mit allen Vorsichtsmassregeln durchschnitten und die Wunde wieder vernäht. Als derselbe sich am folgenden Tage ganz wohl befand, wurde die Erregbarkeit des Nervus ischiadicus derselben Seite geprüft, und es fand sich auch hier, dass die Oeffnungsuckung bei vollständiger Einschaltung der Widerstände des Rheochords fehlte.

Diese Versuche wurden dann später noch dadurch modificirt, dass die sensibeln Wurzeln beider Seiten durchschnitten wurden. Die Versuche wurden ohne und mit Aufiegen von Eis und auch mit vollständiger Präparation des Schädels und der Wirbelsäule gemacht, und es ergab sich hier dasselbe Resultat.

Fassen wir nun das Resultat dieser physiologischen Untersuchungen zusammen :

1. An dem mit dem Centralorgan verbundenen Nerven tritt die Oeffnungszuckung des aufsteigenden Stromes bedeutend späterauf, als an dem rom Centralorgan getrennten.

2. Durch $15-30$ minutenlanges Auflegen einer Kältemischung auf die Wirbelsäule tritt die Oeffnungsuckung noch später auf.

3. Dieselbe Wirkung hat Trennung des Schädels und der Wirbelsäule von allen Theilen mit Ausnahme der zu präfenden Hintersehenkel während der ersten $15-20 \mathrm{Mi}-$ nuten nach der Operation.

4. Die sensibeln Wurzeln können für dieso Thatsachen nicht verantwortich gemacht werden.

Ohne an dieser Stelle weiter auf etwaige Schlüsse aus diesen Resultaten der Untersuchung einzugehen, hebe ich nur das eine her- 
vor, dass in dem mit dem Centralorgan verbundenen motorischen Nerven ständige Einwirkungen sich geltend machen, die sich durch Veränderung der elektrischen Erregbarkeit ausdrücken und in dem vom Centralorgan getrennten Nerven jedenfalls nicht nachweisbar sind.

Ob wir aber diese wohl mit vollstem Recht dem Rückenmark zugeschriebenen Einwirkungen für Tonus und Ernährung der Nerven und Muskeln verantwortlich machen können, lässt sich aus diesen Untersuchungen nicht entscheiden und vielleicht von Seiten der experimentellen Physiologie überhaupt nicht.

Hier beginnt das Gebiet der Pathologie und ihr ist es vielleicht möglich, diese Fragen zum Abschluss zu bringen. Meine eigenen pathologischen Beobachtungen scheinen die physiologischen Resulate zu bestătigen und vielleicht $\mathrm{zu}$ erweitern durch die Thatsache, dass auch am getrennten resp. leistungsunfähigen Nerven des Menschen, bei peripheren Lähmungen. trotz der Schwierigkeit der Untersuchung durch die Hautdecken u. s. w. die Oeffnungszuckung früher anftritt, als an dem mit dem Centralorgan verbundenen.

Bevor ich jedoch näher hierauf eingehe, sei es mir gestattet, einige einleitende Worte vorauszuschicken.

Veränderungen der faradischen und galvanischen Erregbarkeit der Nerven bei den verschiedensten Affectionen sind schon seit langer Zeit Gegenstand der Untersuchung gewesen. Aber erst mit der Einführung einer exacten Lntersuchungsmethode durch Erb konnten genauere und, soweit bis jetzt zu beurtheilen, recht zuverlässige Resultate erhalten werden. Allerdings musste dadurch eine Reihe von Angaben früherer Beobachter als nicht beweisend zuruckgewiesen werden. Hatte man doch durch Nichtberücksichtigung des Leitungswiderstandes der Epidermis nnd der übrigen Gewebe und somit der in den Körper eingeführten Elektricitätsmenge die durch Abnahme der ausserwesentlichen Widerstände bedingten stärkeren Zuckungen bei länger andauernder Reizung der Nerven vielfach einer durch den elektrischen Strom gesteigerten Erregbarkeit dieser zugeschrieben.

So kommt es, dass sicher bewiesene Beobachtungen nur in ganz kleiner Zahl vorhanden sind.

Die erste dieser Art ist die von $\mathrm{Erb}^{*}$ ) zu gleicher Zeit mit der Einführung seiner neven Untersuchungsmethode beschriebene hochgradige Steigerung der faradischen und galvanischen Erregbarkeit sämmtlicher motorischen Nerven des Körpers (mit Ausnahme des Nervus facialis) bei der Tetanie.

*) Dieses Archiv. Band IV. Seite 271. 
Die Steigerung der galvanischen Erregbarkeit charakterisirt sich durch das frühzeitige Auftreten von Kathodenschliessungszuckungen, durch das Erscheinen von Kathodenschliessungstetanus bei verhältnissmässig geringen Stromstärken und das zuwor am Menschen nicht beobachtete Auftreten von Anodenöffnungstetanus.

Auf die aus diesen Ergebnissen der elektrischen Untersuchung gezogenen Schlïsse gehe ich nicht näher ein.

Seit dieser Veröffentlichung Erb's ist die Untersuchung der faradischen und galvanischen Erregbarkeit der Nerven ein häufiger Gegenstand der Forschung gewesen. Abgesehen von der bis zum vollständigen Verlust fortschreitenden Abnahme der faradischen und galvanischen Erregbarkeit der Nerven bei der Degeneration derselben in Folge Trennung vom Centralorgan oder Affection der grauen Vordersäulen des Rückenmarks mit nachfolgender rapider Muskelatrophie und Entartungsreaction, hat sich in einzelnen Fällen von chronischer Myelitis nur eine Steigerung oder Verminderung der Erregbarkeit ohne qualitative Anomalien gefunden. Eine Bedeutung konnte diesem jedenfalls pathologischen Verhalten bis jetzt nicht zugewiesen werden.

Am meisten ist wohl die Tabes dorsalis Gegenstand der elektrischen Untersuchung gewesen. Die einzelnen Angaben der Autoren über ihre Befunde sind ziemlich verschieden. Erb") schliesst aus eigenen Beobachtungen im Vergleich mit den Resultaten anderer Forscher, dass in den früheren Stadien der Tabes eher eine Steigerung, in den späteren eher eine Verminderung der elektrischen Erregbarkeit vorhanden sei. Qualitative Aenderungen der galvanischen Erregbarkeit konnte Erb nicht constatiren; speciell gelang es ihm nie die angeblich vorhandene grössere Neigung zu Oeffnungszuckungen zu finden, so dass für die diagnostische und und prognostische Beurtheilung der Tabes die elektrische Untersuchung bis jetzt ohne wesentliche Bedeutung ist.

In never Zeit hat auch das Verhalten der elektrischen Erregbarkeit der Nerven bei der spastischen Spinalparalyse etwas die Aufmerksamkeit auf sich gelenkt. In einer grossen Anzahl Fälle von dieser Affection fand Erb eine allerdings nur geringgradige Herabsetzung der faradischen und galvanischen Erregbarkeit der Nerven.

Ich hatte Gelegenheit, einen Theil dieser Fälle gleichfalls zu untersuchen und konnte auch in etlichen andern, bis jetzt nicht veröffentlichten Fällen dasselbe Verhalten constatiren.

Auch bei der Halbseitenläsion wurde in mehreren Fällen von

*) Ziemssen, Handbuch, Band XI., II., 2, 176. 
Müller, Joffroy, Solmon und Erb eine Herabsetzung der elektrischen Erregbarkeit nachgewiesen.

Zum Schluss muss ich noch Eines elwähnen, worauf allerdings schon Erb aufmerksam gemacht hat, dass die hie und da erwähnte Behauptung, der Nerv sei bei einer Lähmung faradisch unerregbar gewesen, habe jedoch auf den galvanischen Strom in qualitativ veränderter Weise ( $\mathrm{AnSZ} \geq \mathrm{KSZ}$, beide träge, tonisch) reagirt, jedenfalls auf falscher Beobachtung beruht.

Bis jetzt ist wohl die Angabe Erb's vollständig angenommen, dass schon kurze Zeit nach dem Entstehen der Lähmung ein continuirliches und glejehmässiges Sinken der faradischen und galvanischen Erregbarkeit in Nerven eintritt und zwar ohne irgendwelche qualitative Veränderung derselben. Im Lauf der ersten oder zweiten Woche erlischt dann mit der Degeneration die Erregbarkeit völlig.

In anderen Fällen, bei der sogenanten Mittelform, tritt nur eine unbedeutende Verminderung der faradischen und galvanischen Erregbarkeit ein, die dann mit dem Verschwinden der Lähmung vergeht. Unterdessen tritt langsam die Atrophie und Entartung der Muskeln mit der ausgesprochenen qualitativen Aendernng der elektrischen Erregbarkeit ein und geht bei Heilung ebenso langsam zurück.

Als ich nun bei meinen Untersuchungen ïber einen Einfluss der Centralorgane auf den motorischen Nerven zu dem Resultat gekommen war, dass an dem mit dem Centralorgan verbundenen Nerven die Oeffnungszuckung weit später auftrat, als an dem vom Centralorgan getrennten, lag es nahe, auch am Menschen dieselbe Prüfung zu machen.

Periphere Lähmungen gehören gewiss nicht zu dem seltensten Material der elektro-therapeutischen Abtheilungen; aber sie kommen leider nur allzuoft erst spät zur Untersuchung, wenn der Nerv vollständig degenerirt ist.

In dieser Beziehung haben die Mittelformen für die Untersuchung der Nerven vielleicht einen Vortheil, wenn auch durch die meist fehlende Beobachtung direct nach dem Beginn der Lähmung eine Fehlerquelle gesetzt sein wird, die allerdings dadurch wieder etwas compensirt wird, dass meist die Möglichkeit vorliegt, den Fall bis zur Ausgleichung der Störungen untersuchen zu können.

Der erste Patient, den ich untersuchte, befand sich damals am fünften Tage auf der hiesigen elektro-therapeutischen Station. 


\section{Fall I.}

Carl Claus, 36 Jahre alt, hatte for ungefähr zehn Wochen bei einem Fall den rechten Unterschenkel gebrochen und war in der Klinik zu Giessen behandelt worden. Am 19. Juni wurde er von dort, da er auf den gebrochenen Fuss noch nicht treten konnte, mit Krücken entlassen und befand sich mehrere Tage auf der Reise. Schon bald nach Gebranch der Krücken litt Patient an heftigen Schmerzen im rechten Arm und drei bis vier Tage später bemerkte er beim Anziehen des Rockes, dass der rechte Arm theilweise lahm war, indem die Hand nicht mehr gestreckt werden konnte.

Am 1. Juli wurde folgender Status aufgenommen:

Patient ist ein grosser, krä̈tiger Mann. Beim Anblick fällt sofort auf, dass die rechte Hand in Beagestellung steht, aus der sie nicht dorsalflectirt werden kann. Nur die zwei Endphalangen der Finger können gestreckt werden. Bei dem Versuch den Vorderarm in halber Supination zu beugen, wird nur der Musculus biceps innervirt. Bei gestrecktem Arm mit Fixation des Humerus kann die Hand nicht supinirt werden.

Der gebeugte Arm kann nicht gestreclt werden: keine Contraction des Musculus trioeps.

Ausser etwas Taubheitsgefühl in der Gegend des rechten Daumens keine Sensibilitätsstörung.

Die elektrische Untersnchung ergiebt: Links ist das Radialisgebiet von der Umbeugestelle, von der Achselhöhle und von der Oberschlüsselbeingrube erregbar, rechts nur von der Umbeugestelle. Eine Herabsetzung der Erregbarkeit des peripheren Stiicks des Nervus radialis dexter liess sich nicht constatiren.

Keine Entartungsreaction der Muskeln.

Die Diagnose lantete: Lähmung des Nervus radialis dexter mit Einbegriffensein der Aeste für die M. M. supinator brevis, longus und triceps. Die Läsionsstelle musste sich in der Achselhöhle befinden (Krückenlähmung).

Am folgenden Tage liess sich geringe Herabsetzung der faradischen Erregbarkeit des gelähmten Nerven (um 10 Millimeter Rollenabstand) nachweisen. Galvanisch war der Nery ebonfalls erregbar. KaSZ erfolgte auf beiden Seiten b ei der gleichen Elementenzahl.

In den Muskeln war schon eine deutliche Steigerung der galvanischen Erregbarkeit vorhanden. Es zeigten sich die ersten Anfänge der Entartungsreaction.

Erst am fünften Tage kam ich dazu, den Nervus radialis auch auf seine qualitative Erregbarkeit genauer zu prüfen.

Die Untersuchung ergab:

KaSZ r b. 6 Elem. $12^{\circ}$ Nadelablenkung

$" 1$ b. 8 " $11^{0}$ "


AnSZ r b. 12 Elem. $30^{\circ}$ Nadelablenkung

\begin{tabular}{|c|c|}
\hline,$\quad 1$ b. 12 , & $29^{\circ}$ \\
\hline An OZ r b. $10 \mathrm{EI}$. & $25^{0}$ \\
\hline
\end{tabular}

Es ergab sich also, dass an dem vom Centralorgan getrennten Nerven die AnOZ um $10^{\circ}$ frizher auftrat, als an dem der andern Seite. Damit schien mein am Froschnerven erhaltenes Resultat bestätigt, wenn es sich hier nicht etwa um einen durch unberücksichligte Fehler hervorgerufenen Irrthum handelte, oder vielleicht gar derartige Differenzen sich auch bei Gesunden vorfinden liessen. Um einen Irrthum durch Verrücken der Elektrode konnte es sich nicht handeln; denn die Wiederholung ergab dasselbe Resultat.

Dann war zunächst ein Nerr anf seine qualitative Erregbarkeit geprüft worden, wobei also AnS und AnO in einer Schliessung und Oeffinung untersucht worden waren. Ausserdem hatte sich bei KaSZ und AnSZ zwischen rechts und links $\left(12^{\circ}\right.$ und $11^{\circ}, 30^{\circ}$ und $\left.29^{\circ}\right)$ keine Differenz ergeben. so dass die Erhöhung der Erregbarkeit eigentlich keinem Zweifel mehr unterliegen konnte.

Um aber jeden Irrthum zu vermeiden, dehnte ich meine Untersuchungen auch anf eine grosse Anzahl vollständig gesunder Personen aus und es zeigte sich hier, wie das allerdings schon bekannt, dass die einzelnen Zuckungen auf beiden Seiten bei fast absolut der gleichen Stromstärke auftreten. Ich führe hier nur ein Beispiel an:

K. Schütz, 20 Jahre alt, an den Armen vollständig gesund. Nervus radialis.

$$
\begin{aligned}
& \text { KaSZ r } 8 \text { El. } 10^{\circ} \mathrm{Te} 12 \mathrm{El} .30^{0} \\
& \text { " } 18 \text { El. } 90 \quad \text { 12 El, } 27 \\
& \text { An SZ } 12 \text { El. } 25^{\circ} \\
& \text { " } 112 \mathrm{El} .24^{\circ} \\
& \mathrm{AOZ} \text { r } 12 \mathrm{El} .25^{\circ} \\
& \text {, } 112 \text { El. } 24^{\circ}
\end{aligned}
$$

Ich muss hier dem Verhältniss der AnSZ zur AnOZ noch einige Worte widmen. $\mathrm{Erb}^{*}$ ) führt bei seinen Untersuchungen an, dass im $\mathrm{N}$. radialis die An OZ weit über die AnSZ präralire und früher auftrete. Wenn man durch mehrfache Kathodenschliessungen einige Kräflige Zucliungen im Gebiet des N. radialis ausgelöst hat und nun mit der Anode schliesst und offnet, so fällt es allerdings auf, wie sehr die Anodenöffnungszuckung $\left.{ }^{*}{ }^{*}\right)$ an Stärke diejenige der Schliessung übertrifft.

*) Dieses Arehiv, Band IV. S. 281.

**) Es scheint mir hier am Platz zu sein, auf ein Verhalten der Anodenöffnungszuckungen hinzuweisen, das gewiss schon jedem, der sich mit der Prüfung des Zuckungsgesetzes am Menschen beschäftigt hat, aufgefallen ist: es ist das weit frühere und stärkere Auftreten derselben nach mehreren Kathodenschliessungen oder nach langer Dauer der Anodenschliessung. 
Prüftman aber die Anode allein, indem man mit langsamer Steigung der Stromstärke je dreimal schliesst und öffnet, so zeigt sich, dass die Minimalzuckungen bei AnS und AnO im N. radialis ebenso wie in den andern Nerven bei ziemlich gleicher Stromstärke auftreten, oder die AnSZ vielleicht um ein geringes später. ${ }^{*}$ ) Das erstere Verhältniss zeigt anch unser letzter Untersuchter.

Mit unserm Fall hat allerdirgs dieses Verhältniss der AnSZ zur An OZ direct nichts zu schaffen, da wir ja zum Vergleich die andere Seite zur Verfügung haben und sich zwischen diesen die jedenfalls nicht unbeträchtliche Differenz herausgestellt hatte.

Es konnte somit keinem $Z$ weifel unterliegen, dass es sich in unserm Fall um eine Steigerung der Erregbarkeit für die Anodenöffnungszuckung in dem mit dem Centralorgan nicht in Verbindung stehenden Nervus radialis handelte.

Auch am näehsten Tage liess sich dasselbe Verhältniss constatiren, indem die AnOZ links um 6 Elementespäter auftrat, als rechts. (Da ich den Verlauf noch lange beobachten zu können glaubte, begnügte ich mich mit diesem Resultat.)

Aber schon am 7. Juli, also zwei Tage nach der ersten Untersuchung, war die Steigerung für die Aodenöffnungszuckung nicht mehrnach zuweisen. Patient konnte plötzlich das Radialisgebiet mit Ausnahme des M. triceps und supinator longus (die faradische Erregbarkeit in diesen beiden war schon zuvor erloschen) wieder be wegen, wenn auch die Bewegungen noch äusserst schwach waren und die gestreckte Hand nach kurzer Zeit herabsank.

Die galvanische Untersuchung ergab;

KaSZ r 8 El. $8^{0}$ Te 14 El. 31

$" 16$ El. $14^{\circ}$ " 14 El. 36

An SZ r 14 El. $34^{\circ}$

" $112 \mathrm{El} .28^{\circ}$

An OZ r 12 El. 290

" $114 \mathrm{El} .320$

Oberflächlich könnte es scheinen, als sei diese Veränderung durch Abnahme der Leitungswiderstände bedingt; aber eine genauere Untersuchung ergiebt, dass diese Abnahme nicht die Ursache sein kann.

Ich kann mir dieses Verhalten nicht anders erklären, als dass sich im Nerven ständige immer neu entstehende oder zugeführte Widerstände dem durch die Anodenöffnung gesetzten Reiz entgegenstemmen, die erst durch den mit einer starken Kathodenschliessungszuckung verbundenen Erregungsvorgang oder durch längeres Fliessen des Stromes vernichtet werden.

*) Es kommt übrigens auch vor, dass die An OZ weit später als die AnSZ auftritt, wofür ein späterer Fall als Beleg dienen wird.

Weitere Untersuchungen der qualitativen galvanischen Eriegbarkeit geben vielleicht Anhaltspunkte zur Erklärung dieser Verschiedenheiten. 
Der vor zwei Tagen vorhandene Unterschied war also nicht mehr zu constatiren.

Die Entartungsreaction der Muskeln war aber a uf das deutlichste nachzuweisen. Die AnSZ übertraf die KaSZ entschieden.

Eine kleine Reise hielt mich indessen ab, den Fall die nächsten Tage zu beobachten.

Nach meiner Rückkehr am 23. Juli fand ioh sämmtliche Muskeln mit Ausnahme des M. triceps and supinator longus schon wieder recht kräftig. Die Reizung des Nerven in der Achselhöhle und Oberschlüsselbeingrube ergab eine kräftige Contraction in den wieder horgestellten Muskeln.

Die elektrische Untersuchung ergab disselbe Resultat wie zuvor. Um nicht zu ermüden, führe ich von den fast täglich gemachten Untersuchungen nur einige an.

Am 27. Angust ergab sich

N. radialis KaSZ r 6 El. $4{ }^{0} \mathrm{Te} 12 \mathrm{El} .31^{\circ}$

$\Rightarrow 16 \mathrm{El} .8^{\circ} " 10 \mathrm{El} .28^{\circ}$

AnSZ r $14 \mathrm{EJ} .32^{\circ}$

$\Rightarrow 114 \mathrm{El} .32^{\circ}$

An OZ r 12 El. $26^{\circ}$

$\rightarrow 112 \mathrm{El} .27^{\circ}$

Zugleich war in den wieder willkürlichen Muskeln die Entartungsreaction lange nicht so deutlich ausgesprochen als in dem M. triceps und supinator longus, in denen bei ganz schwachen Stromstärken AnSZ und KaSZ auftraten, die erstere ziemlich überwiegend.

Ziemlich dasselbe Verhalten zeigte sich acht Tage später:

N. radialis KaSZ r 4 El. $3{ }^{\circ}$ Te 10 El. $25^{\circ}$

$" 14 \mathrm{El} .3^{\circ}, 12 \mathrm{El} .26^{\circ}$

An SZ r 10 El. $18^{\circ}$

$\rightarrow 110 \mathrm{El} .16^{\circ}$

$\mathrm{AOZ}$ r $10 \mathrm{El} .18^{\circ}$

, $110 \mathrm{El} .16^{0}$

Die gleichzeitige Prüfung der faradischen Erregbarkeit ergab

b. 10 Elem. und $150 \mathrm{LW}$.
r. 73
1. 65
NA r. $24^{\circ}$
I. $17^{\circ}$

also ebenfalls keine Abweichung rom Normalen.

Die Untersuchung der Vorderarmmuskeln ergab bei $12 \mathrm{El} .14{ }^{\circ} \mathrm{KaSZ}$ und AnSZ, beide von ziemlich gleicher Grösse, träge und tonisch, in M. supinator longus und triceps bei weit geringerer Stromstärke An SZ > KaSZ.

Im Laufe der nächsten Zeit besserte sich die Kraft der Vorderarme immer mehr. Patient konnte trotz der noch gelähmten beiden Muskeln seiner Beschäftigung als Schreiner zum Theil wieder nachgehen.

In den M. M. supinator longus und triceps war die Rückkehr der willkürlichen Beweglichkeit erst am 22. September zu constatiren. 
Zugleich ergab Reizung des Nerven oberhalb der Läsionsstelle (tief in der Achselhöhle und in der Oberschlüsselbeingrube) auch in diesen beiden wieder $\mathrm{Zuckung}$.

Die Entartungsreaction war in beiden noch auf das deutlichste ausgesprochen: An SZ > KaSZ.

Die galvanische Untersuchung der übrigen zuvor gelähmten Muskeln des Vorderarms ergab

bei 10 El. $18^{\circ} \mathrm{KaSZ}$ und AnSZ.

Jetzt aber war die KaSZ weit grösser als die AnSZ.

Da die Motilität sämmtlicher Muskeln wieder vorhanden war, trat Patient am 23. September ans.

Während ich noch mit Untersuchungen des letzten Patienten beschäftigt war, kam noch ein weiterer Fall von Radialislähmung in meine Beobachtung.

\section{Fall II.}

W. Treiber, 50 Jahre alt, bemerkte vor sieben Tagen beim Erwachen, dass die rechte Hand theilweise gelähmt war. Eine Ursache weiss er nicht anzugeben; doch erklärt er auf Befragen, dass er die Nacht auf dem Arm geschlafen habe, wie er das oft zu thun pflege.

Am 28. August wurde folgender Status aufgenommen:

Patient ist ein kräftig gebauter Mann. Beim Anblick zeigte sich die rechte Iland in der für Radialislähmug charakteristischen Stellung.

Die genauere Untersuchung ergiebt Lähmung des Radialisgebietes am Vorderarm mit Einbegriffensein der M. M. supinator longus und brevis. Der M. triceps ist willkürlich gut erregbar.

Eine Sensibilitätsstörung lässt sich nicht constatiren.

Links ist das Radialisgebiet von der Umschlagstelle, von der Achselhöhle und Oberschlüsselbeingrube aus erregbar, rechts nur von der Umschlagstelle; bei Reizung von der Achselhöhle und Oberschlüsselbeingrube aus erfoigt $\mathrm{n}$ ur Contraction des M. triceps, das übrige Radialisgebiet bleibt unerregt.

Diø Diagnose lautete: Lähmung des N. radialis dexter ohne Einbegriffensein des Astes für den M. triceps.

Die Läsionsstelle lag also etwas über der Umschlagstelle unterhalb des für den M. triceps abgehenden Astes.

Die Prüfung der elektrischen Erregbarkeit des Nerven ergab mit dem faradischen Strom:

Rolllenabstand

r 47 - I 54 Nadelablenkung bei $10 \mathrm{El}$. and $150 \mathrm{LW}$.

$$
\text { r } 50 \quad 15^{0}
$$

also keine nachweisbare Veränderung der faradischen Erregbarkeit;

mit dem galvanischen Strom

KaSZ r 14 El. $33^{\circ}$ Te 20 El. $37^{\circ}$

$" 114 \mathrm{El} .21^{\circ} \# 22 \mathrm{El} .38^{\circ}$ 
An SZ r 22 El. $35^{\circ}$

$" 122$ El. $36^{\circ}$

An $O Z$ r 14 El. $23^{\circ}$

" $120 \mathrm{El} 32^{\circ}$ (anfangs $22 \mathrm{El} .34 \%$ ).

Die galvanische Untersuchung ergab somit Herabsetzung der Erregbarkeit für die KaS und deutliche Steigerung für die An 0 um $9{ }^{0}$. Die AnSZ erfolgten beiderseits völlig gleich.

Entartungsreaction liess sich in den gelähmten Muskeln des rechten $\mathrm{N}$. radialis noch nicht nachweisen. handlung.

Patient kam erst am zweiten Tage, am 30. August wieder zur Be-

Die Untersuchung ergab:

N. radialis $\mathrm{KaSZ}$ r 12 El. $16^{\circ} \mathrm{Te} 20 \mathrm{EI} .38^{\circ}$

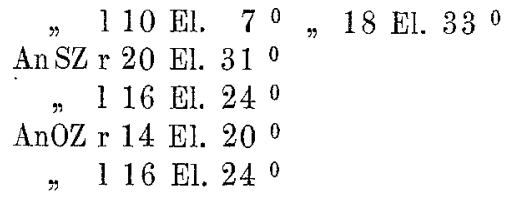

Vergleicht man bei dieser Untersuchung nur die beiden Anodenöffrnungszuckungen, so ist diejenige der rechten Seite allerdings kaum merklich erhöht. Zieht man aber gleichzeitig in Betracht, dass zur Hervorrufung der Ka SZ und An SZ rechts entschieden höhere Stromstärken nothwendig sind als links, also die Erregbarkeit des Nerven für diese beiden herabgesetzt ist, zur Hervorrufung der $\triangle \mathrm{nOZ}$ rechts dagegen eine geringere Stromstärke als links, so lässt sich eine Steigerung der Erregbarkeit für die Anodenöffnung im $r$ echten Radialis nicht verkennen.

Dasselbe Resultat ergiebt auch eine Vergleichung der beiderseitigen AnSZ and AnOZ. Auf der linken Seite treten dieselben bei der gleichen Stromstärke auf, im rechten Radialis dagegen die AnOZ um $11^{\circ}$ früher als die An SZ. 31. August.

Dasselbe Resultat ergab die Cntersuchung am folgenden Tage, am

\begin{tabular}{|c|c|}
\hline N. radialis & 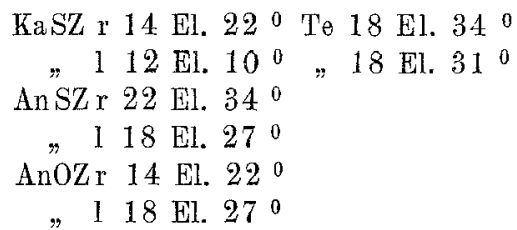 \\
\hline
\end{tabular}

Die Prüfung mit dem faradischen Strom ergielt rechts Zuckung bei einm Rollenabstand von 49 , links von 57 Millimeter.

Ausserdem zeigten sich die ersten Spuren von Entartungreaction, indem rechts bei $18 \mathrm{El} .27^{0}$ neben der noch mehr blitzähnlichen Ka SZ eine träge, tonische AnSZ auftrat, links war bei 18 und 20 El. nur eine blitzähnliche KaSZ zu constatiren. 
Deutlicher war die Entartungsreaction schon am folgenden Tage, dessen Untersuchungsresultat ich noch aus einem andern Grunde hier anführe:

N. radialis KaSZ $\mathrm{r}_{*} 14$ El. $19^{\circ}$ Te 24 El. $39^{\circ}$

$\begin{array}{rrrr}1 & 12 \text { El. } 6^{\circ} \\ \text { An SZ r } & 20 \text { El. } 29^{\circ} \\ \Rightarrow 1 & 18 \text { El. } 25^{\circ} \\ \text { AnOZr } & 16 \text { El. } 21^{\circ} \\ \Rightarrow 1 & 18 \text { El. } 25^{\circ}\end{array}$

Soweit war das Resultat von dem vorigen nicht versehieden, aber bei der Prüfung des KaSTe rechts stellte sich plötzlich bei 20 El. $36^{\circ}$, wobei noch kein Tetanus auftrat, $\mathrm{KaOZ}$ ein.

- Es war somit durch das Sinken der Erregbarkeit für den KaSTe die $\mathrm{KaOZ}$ deutlich geworden.

Die Prüfung der Muskeln ergab rechts bei 18 Elementen eine etwas träge Ka SZ, bei 20 Elementen eine ganz tonische AnSZ.

Zwei Tage später konnte Patient schon wieder ganz minimale Bewegungen im Gebiet der gelähmten Muskeln machen; vor allem war er im Stande die dorsalflectirte Hand mehrere Sekunden in ihrer Stellung zu erhalten.

Dem entsprechend war auch die Herabsetzung der Erregbarkeit für Kathoden- und Aodenschliessung nicht mehr so dentlich ausgesprochen und damit liess sich auch das verhältnissmässig frühe $A$ uftreten der AnOZ nicht mehr constatiren.

Die Untersuchung am 3. September ergab:

Rollenabstand b. $10 \mathrm{El}$. und $150 \mathrm{LW}$.

Farad. Str. r. 39 l. 54 - NAbl. r. $4^{0} 1.4^{0}$

Ka SZ r $12 \mathrm{El} .11^{\circ} \mathrm{Te} 22 \mathrm{El} .35^{\circ}$

" $112 \mathrm{El} .66^{\circ}, 20 \mathrm{El} .34^{\circ}$

AnSZ r $18 \mathrm{El} .25^{\circ}$

" $118 \mathrm{El} .21^{\circ}$

$\mathrm{AnOZ}$ r $16 \mathrm{El} .22^{0}$

$" 118$ El. $21^{\circ}$

KaOZ war nicht mehr zu erzielen.

Die Entartungsreaction der Muskeln war deutlich ausgesprochen.

Die Reizung des Nerven in der Achselhöhle und Oberschlüsselbeingrube löste hingegen noch keine Zuckung in den gelähmten Muskeln aus, während die andern Nervenstämme leicht auf den faradischen Strom reagirten.

Die Untersuchung am folgenden Tage ergab ganz das nämliche Resultat. Nur war die Entartungsreaction noch etwas deutlicher ansgesprochen: bei 14 Elementen erfolgte rechts $\mathrm{KaSZ}$ und $\mathrm{AnSZ}$ beide tonisch und träge und zwar überwog die AnSZ etwas über die $\mathrm{KaSZ}$. Patient konnte die Mnskeln schon etwas kräftiger bewegen.

Am 5. September liess sich in der elektrischen Erregbarkeit der N. radiales keine Differenz mehr finden. 


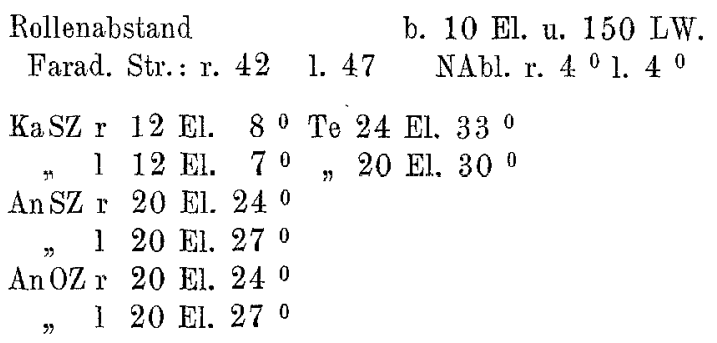

Bei Reizung der Nuskeln erfolgte bei 16 El. $12^{\circ} \mathrm{KaSZ}$ und AnSZ. Doch überwog nunmehr die KaSZ schon wieder etwas über die AnSZ. Oberhalb der Läsionsstelle liess sich vom $\mathrm{N}$. radialis aus noch keine Zuckung in den Muskeln auslösen.

Dasselbe Resultat ergaben die Untersuchungen in den nächsten Tagen. Die Bewegungen blieben lange schwach und nur sohr langsam liäftigten sich die Muskeln.

Erst am 22. September, nachdem Patient allerdings einige Tage nicht zur Behandlung gekommen war, liess sich von dex Achselhöhle und der Obersehlizsslbeingrube aus Contraction in den zuvor gelähmten Muskeln erzielen.

Die Entartungsreaction war schon wieder im Abnehmen begriffen. Wonigstens erfolgte erst bei $14 \mathrm{El} .18^{\circ} \mathrm{KaSZ}$ und AnSZ, beide träge und tonisch, aber KaSZ schon wieder überwiegend.

Patient wurde auf seinen Wunsch aus der Behandlung entlassen.

Ohne hier auf Schlüsse aus diesen Beobachtungen einzugehen, hebe ich nur das hervor, dass sich in beiden Fällen von peripherer Lähmung eine Steigerung der Erregbarkeit für die Anodenöffnung ergab. Und zwar stammen die Beobachtungen des ersten Falls rom zwölften und dreizehnten, die des zweiten vom siebenten bis zwölften Tag.

Bei beiden Fällen war diese qualitative Veränderung mit dem Verschwinden der Lähmung nicht mehr zu constatiren.

Wie das Verhalten dieser Veränderung bei den schweren Formen von Lähmung, bei denen die Erregbarkeit der Nerven selır bald verschwindet, sein wird, lässt sich natürlich nicht sagen.

In älteren Fällen, in denen die elektrische Erregbarkeit des Nerven schon sehr beträchtlich herabgesetzt ist, lässt sich diese Steigerung der Erregbarkeit für die Anodenöffnung jedenfalls nicht mehr nachweisen, wie das ein kürzlich beobachteter Fall beweist: 


\section{F a 11 III.}

Margaretha Schmidt, 52 Jahre alt, hat schon seit einer Reihe von Jahren eine Geschwulst in der rechten Achselhöhle, die bis zum Juni d. J. jedoch kaum gewachsen ist und auch nur wenig Beschwerden verursachte. Seit dieser Zeit hat die Geschwulst ausserordentlich zugenommen.

Vor etwa acht Wochen bemerkte Patientin, dass eine Lähmang an der rechten Hand hinzngekommen war, diesich bis jetzt nicht gebessert hat.

Die Untersuchung ergiebt ausser der Geschwulst in der rechten Achselhöhle, die nahezu die Grösse eines kleinen Kindskopfes hat, eine Lähmung des Nervus radialis dexter mit Einbegriffensein der Aeste für die M. M. supinator longus, brevis und triceps.

Die elektrische Erregbarkeit des N. radialis dexter war bedeutend herabgesetzt.

In den gelähmten Muskeln war deutliche Entartungsreaction nachweisbar.

Die genauere elektrische Untersuchung des Nerven ergiebt: Rollenabstand b. 12 El. u. $150 \mathrm{LW}$.

Farad. Str.: r. 3 1. 11; NAbl. r. $3^{0} 1.2^{0}$

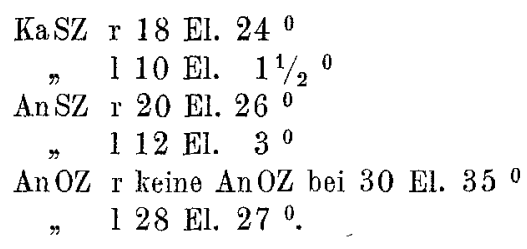

Es liess sich also bei 30 El. $35^{\circ}$ keine An OZ erzielen. Im ersten Augenblick sehien mir diese Thatsache gegen eine Verallgemeinerung meines Befundes bei den vorhergehenden Lähmungen zu sprechen. Berücksichtigt man aber, dass links die AnOZ um $24^{\circ}$ später auftritt, als die AnSZ und fügt diese Differenz der rechts zur AnSZ nöthigen Stromstärke hinzu, so ergiebt sich, dass bei gleichmässigem Sinken der Erregbarkeit für sämmtliche Stromqualitäten die $A n O Z$ rechts bei $50^{\circ}$ hätte auftreten müssen, während sie nur bei $35^{0}$ nicht nachweisbar war.

Im Uebrigen hat der Fall noch dadurch ein Interesse, dass im Nervus radialis die AnOZ um so sehr viel später auftritt, als die AnSZ. Ich habe schon oben vorgreifend darauf hingewiesen.

Diese Ergebnisse der Untersuchungen der qualitativen elektrischen Erregbarkeit bei peripheren Lähmungen waren die Veranlassung zu weitern Untersuchungen derselben bei andern Affectionen. 
Wenn ich einige dieser Beobachtungen gleich hier anschliesse, so geschieht es aus dem Grunde, weil sie als Gegensatz zu den obigen Fällen gewiss einiges Interesse in Anspruch $\mathrm{zu}$ nehmen berechtigt sind.

Es sind dieses Fälle, bei denen weder Lähmung noch Entartung der Nerven und Muskeln statt hat und doch durch die unterbrochene oder nicht genügende Function des Muskels secundäre Atrophie desselben eintritt und mit ihr jene Veränderungen an den Gefässen, die dann auch mehr oder minder das Binde- und Fettgewebe in ihrer Ernährung beeinflussen.

Diese nach den verschiedenen Gelenkaffectionen in den Muskeln der Extremitäten auftretenden und hier so bedeutungsvollen Veränderungen charakterisiren sich im Gegensatz zu den Lähmungen mit Entartungsreaction hauptsächlich durch einfache Atrophie und Verschmälerung der Muskelfasern und erst spät tritt eine Ablagerung von feinsten Körnern zwischen den Fibrillen auf.

Allerdings hat das rasche Auftreten dieser Atrophie nach Entzündung der Gelenke im Gegensatz zu den kaum oder wenigstens lange nicht nachweisbaren Veränderungen bei blosser Inactivität der Muskeln, wie sie als Folge von Gehirnaffectionen vorkommt, in neuerer Zeit etliche Forscher veranlasst, die Ursache hiervon noch in einem weitern Moment zu suchen. James Paget*) hat wohl zuerst die Affection als eine reflectorische aufgefasst, bedingt durch die den sensibeln Nerven übermittelten Erregungen. Weitere Momente hat Valtat,**) der anf Anregung ron Le Fort klinisch und experimentell über diese Frage arbeitete, gegen die Auffassung der Atrophie als einer nur durch Inactivität bedingten beigebracht. Nachdem er das ausserordentlich rasche Auftreten derselben nach Affectionen der Gelenke hervorgehoben, spricht er sich gegen die eine Ansicht aus, dass die Atrophie einem Weiterkriechen des entzündlichen Processes in die Mukeln ihre Entstehung verdanke und führt als Beweis hierfür an, dass der ergriffene Muskel gleichmässig in seiner ganzen Länge atrophire.

Auch eine entzündliche Affection der Nerven und des Rückenmarks schliesst er aus und kommt zum Schluss zu der Ansicht, dass die Atrophie durch einen gleichen Vorgang zu Stande komme, wie einige reflectorische Erscheinungen.

*) Paget, Leçons de clinique chirurgicale, traduction par Petit.

**) Valtat, de l'atrophie musculaire, Paris 1877. 
Dass es sich ja bei dieser Affection nicht um die gewöhnliche durch Auflıören des Einflusses Seitens der Ganglienzellen der grauen Vordersäulen bedingte-trophische Störung handeln kann, beweist das Fehlen der charakteristischen Entartungsreaction in den Muskeln.

Dem entsprechend ist auch das Verhalten der weder gelähmten noch degenerirten Nerven, das sich durch einfache Abnahme der elektrischen Erregbarkeit charakterisirt.

Da für die jetzige Frage nur die elektrischen Untersuchungen von Interesse sind, so gebe ich die Krankengeschichten in möglichst kurzem Abriss.

\section{Fall I.}

Jacob Keller, 38 Jahre alt. als Kind von 12 Jahren an einer linlisseitigen Kniegelenksentzündung erkrankt, wurde damals ein Jahr lang in der Kliuik des Prof. Chelius behandelt und mit steifem Knie entlassen.

Seitdem geht Patient fast stets mit Stöcken und seit etwa einem Jahr mit Krücken, wofür ex als Grund ein Kürzerwerden des linken Beins angiebt.

Die Untersuchung ergab nahezu vollständige Ankylose des linken Kniegelenks in ziemlich stumpfem Winkel, Atrophie und Parese des Musculus quadriceps sinister, während die Beuger des Unterschenkels in mässiger Contractur standen. Auch die linke Wade und das ganze Gebiet des linken Nervus peronaeus waren atrophisch und paretisch. Der Fuss stand etwas in Spitzfussstellung. Das linke Bein war bein Stehen etwas kürzer als das rechte, jedenfalls durch die Beugung in Kuie bewirkt. Kein Zurückbleiben der Knochen im Wachsthum.

Die Nerven waren faradisch und galvanisch erregbar.

Keine Entartungsreaction der Muskeln.

Die Untersuchung der qualitativen Erregbarkeit der Nerven ergab:

Nervus cruralis.

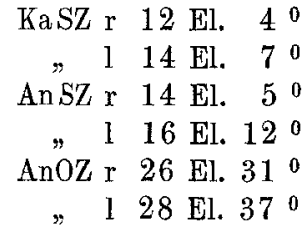

also Herabsetzung der Erregbarkeit für sämmtliche Stromqualitäten links.

Nervus peronaeus

KaSZ r 10 El. $4^{0}$ Te 22 El. $31^{0}$

" $116 \mathrm{El} .15^{\circ} \mathrm{Te}$ nicht zu erzielen

AnSZ r $12 \mathrm{El} .70$

$" 120 \mathrm{El} .27^{\circ}$ 
also ebenfalls eine Herabsetzung der Erregbarkeit für sämmtliche Stromqualitäten links.

Gehen wir auf die Resultate dieser Untersuchung etwas näher ein.

In einem hänfigen und wohl charakterisirten Krankheitsbild finden wir zu einer Zeit, in der das acute Stadium abgelaufen, ein bestimmtes Verhalten der elektrischen Erregbarkeit, ausgezeichnet im Gegensatz zu andern Formen durch eine Abnahme der elektrischen Erregbarkeit der Nerven für sämmtliche Stromqualitäten.

Wenn es anch vielleicht verfrüht ist, aus wenigen derartigen Fällen über die Ursache und das Wesen des pathologischen Vorgangs bei dieser Affection bestimmte Schlüsse zu ziehen, so drängen sich doch aus dem elektrischen Befund einige Fragen auf, deren Beantwortung für die Lehre von der Muskelatrophie nach Gelenkerkrankungen nicht ohne Bedeutung zu sein scheint.

Dass die Herabsetzung der elektrischen Erregbarkeit der Nerven ihre Ursache in der Atrophie und Verschmälerung der Muskelfasern hat, könnte aus dem Grunde wahrscheinlich erscheinen, weil ja, wie bekannt, die Ernährung des Nerven theilweise vom Muskel oder wenigstens von der motorischen Endplatte im Muskel besorgt wird. Aber gegen diese Anschauung, die zugleich die Erklärung der Erscheinungen durch blosse Inactivität zulässt, spricht die Thatsache, dass unter geeigneter Behandlung in den Nerven sehr früh normales Verhalten der elektrischen Erregbarkeit eintritt, während von einer Ausgleichung der Störung in den Muskeln noch lange keine Rede ist.

Auch eine zweite mögliche Annahme, dass die Atrophie ihre Ursache in der Erregbarkeitsveränderung des Nerven hat, würde insofern mit der Anschauung ïbereinstimmen, dass einzig der Nichtgebranch genügt, um Atrophie hervorzurufen, als ja dieser zunächst die Erregbarkeitsverminderung in den Nerven hervorrufen könnte, worauf die Störung in den Muskeln folgt. Diese Annahme wird durch das Verhalten gelähmter Theile bei cerebralen Affectionen bedenklich erschüttert und es bleibt als die wahrscheinlichste nur die dritte Möglichkeit, dass eine gemeinschaftliche Ursache die Störung in den Nerven und den Muskeln bedingt. Diese Ursache haben wir dann wohl in einer Hemmung der Thätigkeit des Rückenmarks zu suchen, hervorgerufen durch die Seitens der sensibeln Fasern zugeführten starken Erregungen.

Dass diese Anschaunng, die mit der Ansicht von Paget und Valtat von einer reflectorischen Atrophie also vollständig übereinstimmt, durch eine grosse Anzahl Analoga in der Physiologie gestützt wird, brauche ich nicht hinzuzufügen. 
Die verminderte Beeinflussung Seitens der Centralorgane bedingte also dann zugleich die veränderte Erregbarkeit der motorischen Nerven und die Atrophie der Muskeln. Damit tritt aber zugleich die Frage auf, ob sich nicht parallel den hemmenden Einflüssen in den Centralorganen die elektrische Erregbarkeit der Nerven verschieden gestaltet, je nachdem die Untersuchung während des Fortschreitens oder der Besserung der Atrophie statt findet. Allerdings wird das acute Stadium schon durch die Schmerzhaftjgkeit der Gelenkaffection bedingt nur schwer eine Untersuchung gestatten und wir werden in den meisten Fällen uns mit derjenigen Form begnügen müssen, in welcher die Affection stationär geworden ist, vielleicht auch schon zur Heilung tendirt. In ihr finden wir also diese charakteristische Herabsetzung der elektrischen Erregbarkeit für sämmtliche Stromqualitäten.

Diese Atrophien mit Parese nach Gelenkerkrankungen bilden also, wie ich schon früher hervorgehoben habe, in dem elektrischen Befund einen deutlichen Gegensatz zu jenen durch eine Läsionsstelle im Nerven für alle vom Centrum kommenden Erregungen undurchgängigen und mit Entartungsreaction in den Muskeln einhergehenden Paralysen.

Hier finden wir nur eine Herabsetzung für die Schliessung des Stromes, während die Oeffnung*) desselben entschieden einer Steigerung begegnet.

In welchem Causalitätsverhältniss allerdings diese Veränderung der Erregbarkeit in den Nerven zu der mit Entartangsreaction verbundenen Paralyse steht, wird sich nur schwer entscheiden lassen.

Die degenerativen Vorgänge in dem Muskel können jedenfalls nicht als Grund für die abnorme Erregbarkeit des Nerven angesehen werden, da letztere vor der Enartungsreaction nachweisbar durch ihr Auftreten nicht beeinflusst wird und lange vor dem Verschwinden derselben einem normalen Verhalten Platz macht.

Alle Erscheinungen deaten vielmehr darauf hin, dass dieselbe in gewissem Zusammenhang mit dem Rückenmark steht.

*) Ich brauche hier wohl kaum auf die allgemein angenonmene Erklärung des Zuokungsgesetzes am Lebenden aufmerksam zu machen, dass die An SZ und die KaOZ erst secundär dadurch entstehen, dass wir nicht im Stande sind, den Strom in bestimmter Richtung nur durch den Nerven zu leiten, and somit die AnSZ durch die in der Nähe der Anode entstehende ideale Kathode und die KaOZ durch die Anode entsteht.

(Vergleiche Erb, Ueber elektrotonische Erscheinungen, D. Archiv f. klin. Medicin III., 238 and 513,) 
Zunächst hebe ich nur hervor, dass nach dem Verschwinden der Leitungshemmung im Nerven auch die abnorme Erregbarkeit nicht mehr nachweisbar war.

Dann haben meine Versuche am Froschnerven ergeben, dass vom Rückenmark aus eine ständige Einwirkung auf die Nerven und somit wahrscheinlich anch auf die Muskeln statt hat, welche die Erregbarkeit für die Oeffnungszuckung im Nerven herabsetzt.

Damit im Einklang steht auch das Verhalten der Anodenöffnungszuckungen am Menschen, auf das ich schon oben hingewiesen habe.

So verlockend es aber auch ist, eine nachweisbare ständige Einwirkung der Centralorgane oder sagen wir des Rückenmarks auf die motorischen Nerven als den Ausdruck einer dem Rückenmark allgemein zugeschriebenen trophischen und tonischen Thätigkeit zu betrachten, so muss ich mir es doch versagen, auf Grund so weniger Beobachtungen auf diesen Gedanken weiter einzugehen.

Künftige Untersuchungen vielleicht an einer grösseren Anzahl von Fällen, als sie mir zu Gebot stand, sind vielleicht im Stande, diese Frage einer Entscheidung näher zu bringen.

Wenn ich aber diese geringe Zahl von Beobachtungen schon jetzt der Oeffentlichkeit übergebe. so geschieht es zugleich mit der Absicht, zu zeigen, dass diese Untersuchungen, so zeitraubend sie sein mögen, einen reichen Ersatz in unserer Erkenntniss der Vorgänge in den nervösen Organen zu geben im Stande sind, und dass die Neuropathologie schon jetzt diese junge Untersuchungsmethode nicht mehr zu entbehren vermag.

Heidelberg, den 2. December 1877. 